\title{
Determining the Effectiveness of a Salicylic Acid Regimen to Reduce Cytotoxic ROS Associated with Anthracycline Treatment
}

\author{
A. Lekan ${ }^{1}$, C. Queenan ${ }^{1}$, and A. Waldron ${ }^{1}$ \\ ${ }^{1}$ Bergen County Academies, Nano-Structural Imaging Lab, 200 Hackensack Avenue, Hackensack, NJ \\ 07601
}

Anthracyclines are the most commonly used class of chemotherapeutic drugs for the treatment of malignant neoplasias in the United States [1]. However, their clinical usefulness is severely impaired by their dose-dependent cardiotoxicity [2]. Doxorubicin, the first anthracycline developed, is known to be highly cardiotoxic [3]. Epirubicin, another anthracycline, was later developed as a more cardiotolerable alternative to Doxorubicin; however its use still carries a risk of developing acute, dose dependent cardiotoxicity [3,4]. The cardiotoxicity caused by epirubicin can still cause severe complications including cardiomyopathy, heart failure and arrhythmias [5]. It is believed that the mechanism of cardiotoxicity is by generation of highly reactive oxygen species (ROS) which, by means of membrane lipid peroxidation, cause direct damage to cardiomyocyte membranes [6].

Focus over the past few years has centered on antioxidants as a potential solution to cardiotoxicity, with results showing that antioxidants could have cardioprotective effects [6]. Phenolic acids, a subset of the polyphenol family of antioxidants, have seen limited research into their potential to reduce cytotoxic ROS generation associated with anthracycline treatment, and possible cardioprotective effects [1]. The goal of this study was to determine the ability of salicylic acid to act as a cardioprotective agent against epirubicin-induced cardiotoxicity by reduction of ROS production.

The effects of salicylic acid and epirubicin on cell viability were analyzed using MTS assays. The cancer cell lines used in this study correspond to those which epirubicin is used to treat, including lung carcinoma (A549), breast adenocarcinoma (MCF-7) and hepatocellular carcinoma (HepG2). Each cell line was treated for 24 hours separately with salicylic acid and epirubicin treatments in concentrations of $5,10,20,30,40$ and $50 \mu \mathrm{M}$ to establish baseline effects on cell viability. Combination treatments were then studied, where as cells were first pre-treated with salicylic acid for 2 hours and then epirubicin at a $50 \mu \mathrm{M}$ concentration was added and left to incubate for another 24 hours. Results illustrated no significant $(\mathrm{p}<0.05)$ increase in cellular viability after pre-treatment with salicylic acid.

To measure suppression of ROS, a luminescent ROSGLO assay was performed. Once again, a combination treatment was performed on each cell line with ROS suppression then being measured. Results illustrated statistically significant $(\mathrm{p}<0.05)$ suppression of ROS production in cells pre-treated with salicylic acid (Figure 1). Qualitative analysis was then conducted using fluorescence microscopy to analyze ROS production. Cells were treated with a combination treatment, and then stained with CellROX deep green reagent. Decreased fluorescence was seen in samples pre-treated for 2 hours with salicylic acid, indicating a decrease in ROS production (Figure 2). Thus, ROS assay results were verified. Overall, salicylic acid shows promise as an effective treatment to suppress reactive oxygen species generation and prevent cardiotoxicity.

References

[1] MS Horenstein, et al., Mol Genet Metab., Vol.71 (2000) 436-444. 
[2] G. Minotti, et al., Pharmacological Reviews, Vol.56 No.2 (2004) 185-229.

[3] J. M. Scott, et al., Circulation, Vol.124 No.5 (2011) 642-650.

[4] LA Smith, et al., BMC Cancer, Vol.10 (2010) 337.

[5] ET Yeh, et al., Circulation, Vol.109 (2004) 3122-3131.

[6] X Xu, et al., J Pharmacol Exp. Ther., Vol.341 (2012) 183-195.

[7] The authors would like to thank the administration of the Bergen County Technical School District and the Bergen County Academies for their continued support of the research program.

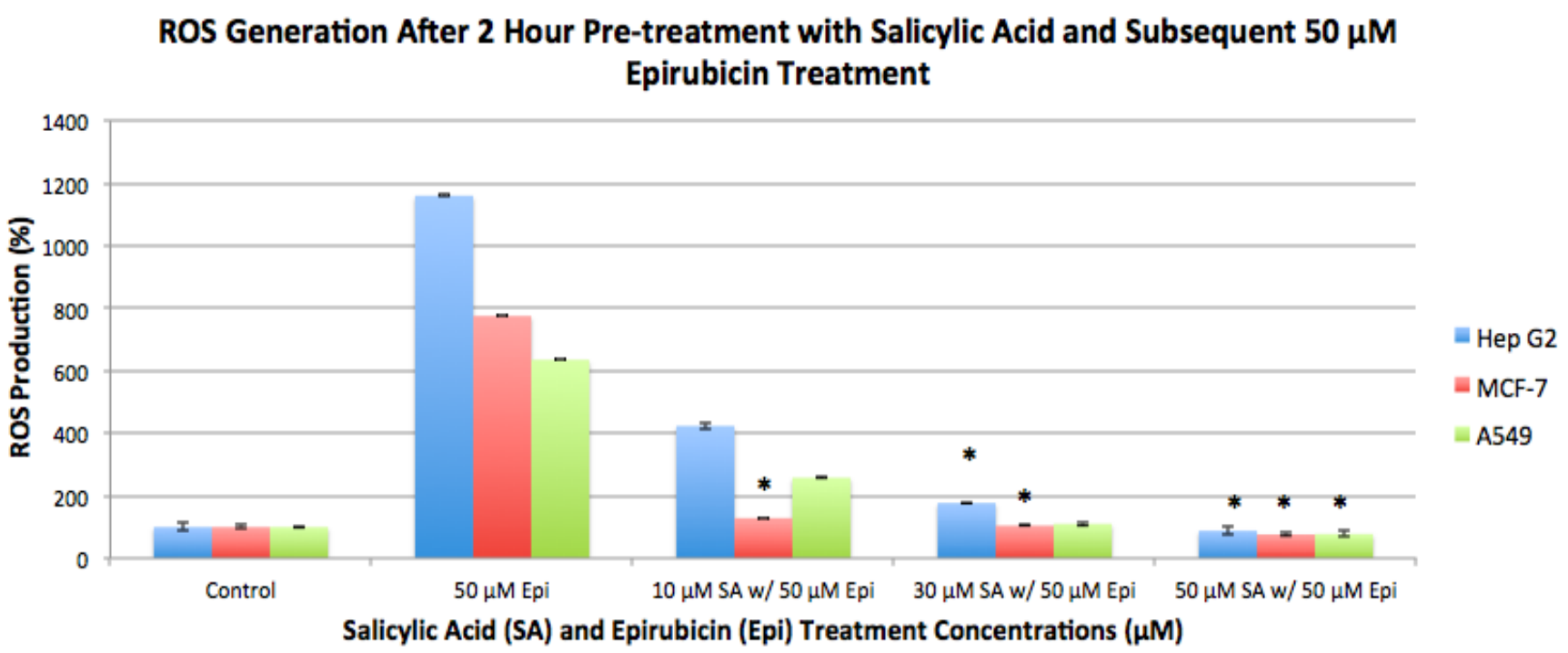

Figure 1: ROS levels in HepG2 (blue), MCF-7 (red) and A549 (green) cells pre-treated with salicylic acid for 2 hours, then treated with $50 \mu \mathrm{M}$ Epirubicin for 24 hours. Data is represented as mean \pm SD $(\mathrm{n}=3)$. Asterisk indicates statistical significance as compared to control $(\mathrm{p}<0.05)$.
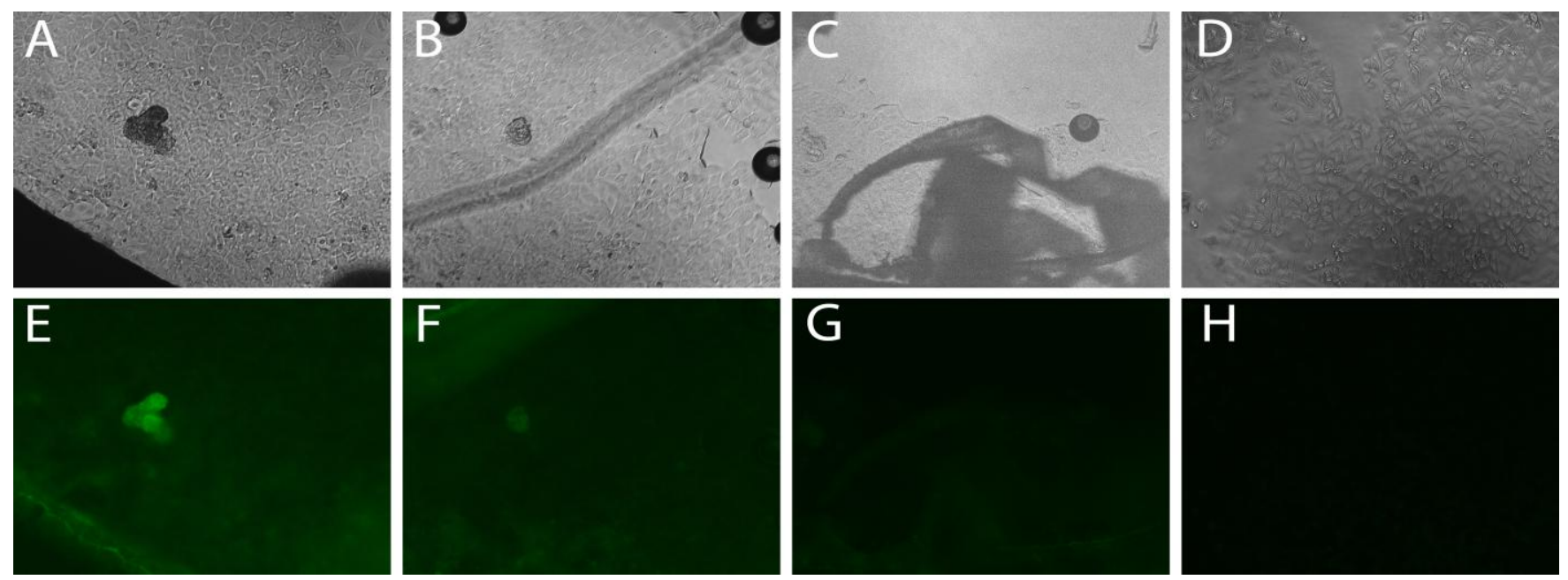

Figure 2: Fluorescent and brightfield microscopy images of MCF-7 cells treated with salicylic acid (SA) and Epirubicin (Epi) and stained with CellROX fluorogenic probe to measure oxidative stress.

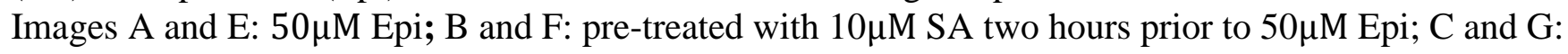
$30 \mu \mathrm{M}$ SA two hours prior to $50 \mu \mathrm{M}$ Epi; D and H: $50 \mu \mathrm{M}$ SA two hours prior to $50 \mu \mathrm{M}$ Epi. All images acquired at 100X magnification. Brighter fluorescence signal indicates greater amounts of ROS present. 